\title{
ON WEIGHTED FIBONACCI AND LUCAS SUMS
}

\author{
Kiyota Ozeki \\ Faculty of Engineering, Utsunomiya University, 7-1-2 Yotoh Utsunomiya, Japan \\ (Submitted May 2002)
}

\section{INTRODUCTION}

T. Koshy wrote a fascinating book [2] on Fibonacci and Lucas numbers. The following summation formulas are well known. Let $S(m):=\sum_{j=1}^{n} j^{m} F_{j}$ and $T(m):=\sum_{j=1}^{n} j^{m} L_{j}$.

$$
\begin{aligned}
& S(0)=F_{n+2}-1 \\
& T(0)=L_{n+2}-3 \\
& S(1)=(n+1) F_{n+2}-F_{n+4}+2 \\
& T(1)=(n+1) F_{n+2}-F_{n+4}+4 .
\end{aligned}
$$

However these are less known formulas [1,2],

$$
\begin{aligned}
S(2) & =(n+1)^{2} F_{n+2}-(2 n+3) F_{n+4}+2 F_{n+6}-8 \\
T(2) & =(n+1)^{2} L_{n+2}-(2 n+3) L_{n+4}+2 L_{n+6}-18 \\
S(3) & =(n+1)^{3} F_{n+2}-\left(3 n^{2}+9 n+7\right) F_{n+4}+(6 n+12) F_{n+6}-6 F_{n+8}+50 \\
T(3) & =(n+1)^{3} L_{n+2}-\left(3 n^{2}+9 n+7\right) L_{n+4}+(6 n+12) L_{n+6}-6 L_{n+8}+112 \\
S(4) & =(n+1)^{4} F_{n+2}-\left(4 n^{3}+18 n^{2}+28 n+15\right) F_{n+4}+\left(12 n^{2}+48 n+50\right) F_{n+6} \\
& -(24 n+60) F_{n+8}+24 F_{n+10}-416 \\
T(4) & =(n+1)^{4} L_{n+2}-\left(4 n^{3}+18 n^{2}+28 n+15\right) L_{n+4}+\left(12 n^{2}+48 n+50\right) L_{n+6} \\
& -(24 n+60) L_{n+8}+24 L_{n+10}-930 .
\end{aligned}
$$

He mentioned a few interesting properties from these formulas without proof. For example, (1) Both $S(m)$ and $T(m)$ contain $m+2$ terms.

(2) The leading term in $S(m)$ is $(n+1)^{m} F_{n+2}$, and that in $T(m)$ is $(n+1)^{m} L_{n+2}$.

(3) The subscripts in the Fibonacci and Lucas sums increase by 2, while the exponents of $n$ in each coefficient decrease by one.

The aim of this note is to establish summation formulas for $S(m)$ and $T(m)$ explicitly. We use the differential operator method, which is discussed in [1]. First we introduce the operator $\nabla f(x)$ which is defined by

$$
\begin{gathered}
\nabla f(x)=x \frac{d f(x)}{d x} \\
\nabla^{n} f(x)=\nabla\left(\nabla^{n-1} f(x)\right), \nabla^{0} f(x)=f(x) .
\end{gathered}
$$

The following lemma is well known, for example [3], and can be proved by straightforward induction. 
Lemma 1: If $f(x)$ is differentiable then

$$
\nabla^{n} f(x)=\sum_{j=1}^{n} S(n, j) x^{j} f^{(j)}(x)
$$

where $S(n, j)$ is the Stirling numbers of the second kind; they are defined by

$$
\begin{gathered}
x^{n}=\sum_{j=0}^{n} S(n, j)(x)_{j} \\
\text { with }(x)_{j}=x(x-1) \ldots(x-j+1),(x)_{0}=1 .
\end{gathered}
$$

Let $f(x)=\sum_{j=1}^{n} x^{j}$ and $g(x)=\frac{1-x^{n+1}}{1-x}$, where $x \neq 1$. We have

$$
\nabla f(x)=\sum_{j=1}^{n} j x^{j} \text { and } \nabla f(x)=\nabla g(x) .
$$

More generally, we have

$$
\nabla^{m} f(x)=\nabla^{m} g(x)=\sum_{j=1}^{n} j^{m} x^{j}, \quad \text { for } \quad m \geq 1 .
$$

Using the Binet formula for Lucas numbers,

$$
\begin{aligned}
T(m) & =\sum_{j=1}^{n} j^{m} L_{j}=\sum_{j=1}^{n} j^{m}\left(\alpha^{j}+\beta^{j}\right)=\left(\nabla^{m} g(x)\right)_{x=\alpha}+\left(\nabla^{m} g(x)\right)_{x=\beta} \\
& =\left(\nabla^{m}\left(g_{0}(x)-g_{n+1}(x)\right)\right)_{x=\alpha}+\left(\nabla^{m}\left(g_{0}(x)=g_{n+1}(x)\right)\right)_{x=\beta}
\end{aligned}
$$

where $g_{t}(x)=\frac{x^{t}}{1-x}$. Suppose we have a formula for $T(m)$. Since we can obtain $F_{i}$ from $L_{i}$ by changing $\beta^{i}$ to $-\beta^{i}$ and then dividing the difference by $\sqrt{5}$, we can find a formula for $S(m)$ from $T(m)$. We consider $T(m)$ in detail.

\section{CONSTANT TERM}

The $j^{\text {th }}$ derivative of $g_{0}(x)$ is expressed by

By Lemma 1 we have

$$
g_{0}^{(j)}(x)=\frac{j !}{(1-x)^{j+1}}
$$

$$
\nabla^{m} g_{0}(x)=\sum_{j=1}^{m} S(m, j) x^{j} g_{0}^{(j)}(x)=\sum_{j=1}^{m} \frac{j ! S(m, j) x^{j}}{(1-x)^{j+1}} .
$$

Since $\frac{1}{1-\alpha}=-\alpha$ and $\frac{1}{1-\beta}=-\beta$, we have the constant term of $T(m)$,

$$
\left(\nabla^{m} g_{0}(x)\right)_{x=\alpha}+\left(\nabla^{m} g_{0}(x)\right)_{x=\beta}=\sum_{j=1}^{m}(-1)^{j+1} j ! S(m, j) L_{2 j+1} .
$$




\section{GENERAL TERM}

Consider the general term for $T(m)$,

$$
\begin{aligned}
\nabla^{m} g_{n+1}(x) & =\sum_{j=1}^{m} S(m, j) x^{j} g_{n+1}^{(j)}(x) \\
& =\sum_{j=1}^{m} S(m, j) x^{j} \sum_{i=0}^{j} i !\left(\begin{array}{c}
j \\
i
\end{array}\right) \frac{\left(x^{n+1}\right)^{(j-i)}}{(1-x)^{i+1}} \\
& =\sum_{j=1}^{m} S(m, j) x^{j} \sum_{i=0}^{j} i !(n+1)_{j-1}\left(\begin{array}{c}
j \\
i
\end{array}\right) \frac{x^{n+i-j+1}}{(1-x)^{i+1}} .
\end{aligned}
$$

So we have

$$
\left(\nabla^{m} g_{n+1}(x)\right)_{x=\alpha}+\left(\nabla^{m} g_{n+1}(x)\right)_{x=\beta}
$$

$$
\begin{aligned}
& =\sum_{j=1}^{m} S(m, j)\left\{\sum_{i=0}^{j-1}(-1)^{i+1} i !(n+1)_{j-i}\left(\begin{array}{l}
j \\
i
\end{array}\right) L_{n+2 i+2}\right\}+\sum_{j=1}^{m}(-1)^{j+1} j ! S(m, j) L_{n+2 j+2} \\
& =-S(m, 1)(n+1)_{1}\left(\begin{array}{l}
1 \\
0
\end{array}\right) L_{n+2} \\
& +\left\{-S(m, 2)(n+1)_{2}\left(\begin{array}{l}
2 \\
0
\end{array}\right) L_{n+2}+1 ! S(m, 2)(n+1)_{1}\left(\begin{array}{l}
2 \\
1
\end{array}\right) L_{n+4}\right\} \\
& +\left\{-S(m, 3)(n+1)_{3}\left(\begin{array}{l}
3 \\
0
\end{array}\right) L_{n+2}+1 ! S(m, 3)(n+1)_{2}\left(\begin{array}{l}
3 \\
1
\end{array}\right) L_{n+4}\right. \\
& \left.-2 ! S(m, 3)(n+1)_{1}\left(\begin{array}{l}
3 \\
2
\end{array}\right) L_{n+6}\right\}+ \\
& +\left\{-S(m, m)(n+1)_{m}\left(\begin{array}{c}
m \\
0
\end{array}\right) L_{n+2}+1 ! S(m, m)(n+1)_{m-1}\left(\begin{array}{c}
m \\
1
\end{array}\right) L_{n+4}+\ldots\right. \\
& \left.\cdots+(-1)^{m}(m-1) ! S(m, m)(n+1)_{1}\left(\begin{array}{c}
m \\
m-1
\end{array}\right) L_{n+2 m}\right\} \\
& +1 ! S(m, 1) L_{n+4}-2 ! S(m, 2) L_{n+6}+3 ! S(m, 3) L_{n+8}+\ldots \\
& \cdots+(-1)^{m}(m-1) ! S(m, m-1) L_{n+2 m}+(-1)^{m+1} m ! S(m, m) L_{n+2 m+2} \text {. }
\end{aligned}
$$


Rearranging terms vertically we have

$$
\begin{aligned}
& \left(\nabla^{m} g_{n+1}(x)\right)_{x=\alpha}+\left(\nabla^{m} g_{n+1}(x)\right)_{x=\beta} \\
& =\left(-\sum_{j=1}^{m} S(m, j)(n+1)_{j}\right) L_{n+2} \\
& +\sum_{t=2}^{m}\left\{\sum_{j=t}^{m}(-1)^{t}(t-1) !\left(\begin{array}{c}
j \\
t-1
\end{array}\right)(n+1)_{j-t+1} S(m, j)+(-1)^{t}(t-1) ! S(m, t-1)\right\} L_{n+2 t} \\
& +(-1)^{m+1} m ! S(m, m) L_{n+2 m+2} .
\end{aligned}
$$

We immediately have

Theorem 1: For $m \geq 2$

$$
\begin{gathered}
\sum_{j=1}^{n} j^{m} L_{j}=(n+1)^{m} L_{n+2} \\
-\sum_{t=2}^{m}\left\{\sum_{j=t}^{m}(-1)^{t}(t-1) !\left(\begin{array}{c}
j \\
t-1
\end{array}\right)(n+1)_{j-t+1} S(m, j)+(-1)^{t}(t-1) ! S(m, t-1)\right\} L_{n+2 t} \\
+(-1)^{m} m ! L_{n+2 m+2}+\sum_{j=1}^{m}(-1)^{j+1} j ! S(m, j) L_{2 j+1} .
\end{gathered}
$$

Replacing $L_{j}$ with $F_{j}$, yields

Theorem 2: For $m \geq 2$

$$
\begin{gathered}
\sum_{j=1}^{n} j^{m} F_{j}=(n+1)^{m} F_{n+2} \\
-\sum_{t=2}^{m}\left\{\sum_{j=t}^{m}(-1)^{t}(t-1) !\left(\begin{array}{c}
j \\
t-1
\end{array}\right)(n+1)_{j-t+1} S(m, j)+(-1)^{t}(t-1) ! S(m, t-1)\right\} F_{n+2 t} \\
+(-1)^{m} m ! F_{n+2 m+2}+\sum_{j=1}^{m}(-1)^{j+1} j ! S(m, j) F_{2 j+1} .
\end{gathered}
$$

\section{REFERENCES}

[1] N. Gauthier. "Fibonacci Sums of the Type $\sum r^{m} F_{m}$." The Mathematical Gazette 79 (1995): 364-367.

[2] T. Koshy. Fibonacci and Lucas Numbers with Applications. A Wiley-Interscience Publication, 2001.

[3] J. Riordan. Combinatorial Identities. Robert E. Krieger Publishing Company, 1979.

AMS Classification Numbers: 11B39, 11B73

\section{四四}

